\title{
Gefangen zwischen den «fünf Fingern der linken Hand»?
}

\section{Eine Streitschrift zur Geschichte der Entpolitisierung am Psychoanalytischen Seminar Zürich ${ }^{1}$ \\ Markus Weilenmann (Rüschlikon)}

Zusammenfassung: Der Artikel geht aufdie Entstehungsgeschichte des Psychoanalytischen Seminars Zürich (PSZ) ein, problematisiert die am PSZ verbreitete Neigung, Politik mit reiner Parteipolitik zu verwechseln und institutionelle Regelungen als etwas dem Wesen der Psychoanalyse Fremdes darzustellen. Er skizziert fünf verschiedene ideologische Verengungen, in die das Seminar hinein geraten ist und die einer inhaltlichen Positionierung des Psychoanalytischen Seminars in der Öffentlichkeit im Wege stehen. Er rät zu einem Überdenken der Absichtserklärung, zu einem neuen Zugang zum Verhältnis Psychoanalyse - Politik und zum Aufbau eines qualifizierten gesellschaftswissenschaftlichen Lehrangebots.

Schlüsselwörter: Psychoanalyse und Politik, Psychoanalyse und Gesellschaft, Parteilichkeit, linke Identität und Psychoanalyse, Institutionsgeschichte.

\section{$1 \quad$ Einführung}

Spätestens seit jenem denkwürdigen IPA-Kongress in Rom (1969), als die so genannte Plattform in der Römer Trattoria «Carlino al Panorama» einen Gegenkongress organisierte mit dem Ziel, die Art wie Psychoanalyse verwaltet wird, in Frage zu stellen und die bestehenden Formen ihrer institutionellen Organisation neu zu überdenken, gehören Fragen zum Verhältnis von Psychoanalyse und Politik, Psychoanalyse und Gesellschaft, Parteilichkeit und nicht zuletzt auch die Frage des «Links-Seins» zur Selbstdefinition und zur Geschichte des Psychoanalytischen Seminars Zürich. Seither sind unzählige Papiere verfasst, Aufsätze und Bücher publiziert, Vorträge gehalten, Kurse durchgeführt und Standpunkte und Meinungen ausgetauscht worden, die sich auf das kulturkritische Potential der Psychoanalyse berufen, die die Radikalität des psychoanalytischen Denkens hervorheben, die Ausbildungsfragen in rigideren oder offeneren Strukturen aufgreifen und die sich der Institutionalisierungsthematik- besonders im Zusammenhang mit dem «Aufstiegund 
Abfall des Psychoanalytischen Seminars Zürich» (Kurz 1993) - annehmen. Insofern mag es vielleicht erstaunen, dass Emilio Modena am Ende seines Artikels über die Geschichte des selbstverwalteten PSZ zu Recht festhält, «dass, übers Ganze gesehen, eine Entpolitisierung stattgefunden hat, zwar nicht in Bezug aufStandespolitik, da ist in den letzten Jahren sehr viel geleistet worden (...) (aber in Bezug auf) die größeren Zusammenhänge» (Modena, in dieser Nummer; Hervorhebung im Text).

Allerdings ist Modenas Feststellung keineswegs neu: Bereits vor bald 30 Jahren, im Journal Nr. 1, machten die «Frösche» ${ }^{2}$ ihrem Frust über die Defacto-Organisation der «Tellstrasse» Luft und skizzierten ein noch immer lesenswertes Bild über die Probleme der Ausbildung, der inneren Machtzirkel und Selbstinszenierungen, der Technik, sich an der «Tellstrasse» zu etablieren, des Anschaffens von Klienten, der noch heute aktuellen Klungeleien und anderem mehr, um schließlich festzustellen, dass auf institutioneller Ebene «die Diskrepanz zwischen Anspruch und Realität ihren Ausdruck im Mangel an gesellschaftswissenschaftlichen Lehrangeboten (findet/MW), im völligen Fehlen von gemeinsamer Forschung, welche die Verbindung zwischen Psychoanalyse und Gesellschaftskritik herstellen könnte und im Mangel an Versuchen, gemeinsame theoretische Bezugsrahmen zu erarbeiten»(1980: 6). Ich teile diese Auffassung auch mehr als 25 Jahre später und möchte in meinen Ausführungen auf einige Sackgassen hinweisen, in die sich das PSZ verwickelt hat und die dazu geführt haben, dass Artikel, die vor 20, 25 oder gar 30 Jahren geschrieben wurden, dem interessierten Leser zum Teil noch heute so aktuell erscheinen, als ob es gestern gewesen sei. Diese Zeitlosigkeit macht stutzig.

Ich denke, die beklagte Entpolitisierung ist Symptom eines Problems, das tiefer sitzt: Es ist dem PSZ in all den Jahren nicht recht gelungen, die politische bzw. gesellschaftliche Dimension des prekären Verhältnisses Psychoanalyse-Politik bzw. Psychoanalyse - Gesellschaft lebendig zu halten. Das Spiel von Rede und Gegenrede beschränkte sich häufig entweder auf die institutionsgeschichtliche Dimension des PSZ - sei es, dass irgendwelche Fakten unterschiedlich dargestellt oder interpretiert wurden -, oder auf die psychologische Dimension - sei es, dass jemandem entgegengehalten wurde, er argumentiere in seinen gesellschaftspolitischen Ausführungen zu ich-psychologisch, zu kleinianisch oder lacanianisch, oder sei es, dass anhand der in analytischen Gesprächen erfassten Details nur die reaktive, subjektiv prägende Seite gesamtgesellschaftlicher Abläufe skizziert wurde.

Mit Blick auf die Geschichte von «Plataforma» fragte Berthold Rothschild (1993: 59), ob «wir uns (tatsächlich) unsere unterschiedlichen Vorstellungen von Psychoanalyse und Politik eingestanden haben?»- eine Frage, die neu aufgegriffen und auf das gesamte PSZ ausgedehnt werden muss. Ich möchte ihm hier nachträg- 
lich antworten, nämlich dass nicht die psychoanalytische wohl aber die politische Dimension dieser Diskussion schon seit langem eine merkwürdig monotone ist; dass der Preis für den Rausschmiss des PSZ aus den Räumen derWaserstrasse sich nicht zuletzt an einerVerengung der politischen Debatte ablesen lässt, die sich nun in Indifferenz zu verlieren scheint; dass über weite Strecken Politik mit Parteilichkeit verwechselt wurde und dass die am PSZ gehandelten ideologischen Versatzstücke sich weniger im Bereich psychoanalytischer Zusammenhänge als in den Bildern über Macht, über den Zwang zur Anpassung, über das gesellschaftlich Bedrohende und das so genannte «Unbehagen in der Kultur» finden lassen; womit die Frage des «Links-Seins» zu einem zunehmend beengenderen und auch inhaltsleereren Korsett wurde, das so schlecht zur Weite des psychoanalytischen Denkens passt.

ImZusammenhang mit dem internationalenVernetzungskongress in Mailand fasste Berthold Rothschild die vorhandenen Tendenzen der politischen Identität von Psychoanalytiker/inne/n im Bild der «fünf Finger der linken Hand» (1988: 8) zusammen $^{3}$. Hier komme ich auf dieses Bild zurück, weil es sich gleichzeitig dazu eignet, den Grad an ideologischer Verengung in einem eingängigen Bild zusammenzufassen. Gefangen zwischen den fünf Fingern der linken Hand

, scheint sich das fortwährende Auseinanderdividieren zwischen Individuum und Staat/ Gesellschaft bzw. Subjekt und Kultur wie ein Automatismus eingespielt zu haben;

, bleibt die Gleichsetzung (oder ist es eine Verwechslung?) von Politik und Parteilichkeit noch heute weitgehend unhinterfragt;

, wird über die Angemessenheit des marxistischen Weltbildes, das mit Psychoanalyse verbunden wurde oder werden soll(te), höchst selten debattiert - wenn überhaupt;

, hat bisher noch kein eigentlicher Reflexionsprozess über die politischen Konsequenzen des Legitimationsbedürfnisses einer Institution eingesetzt, die sich aus der bisherigen Verwaltung von Psychoanalyse gelöst hat und die deshalb einer neuen ideologischen Basis bedarf;

, wird die von verschiedener Seite propagierte Teilung der psychoanalytischen Berufsidentität ${ }^{4}$, die sich, bildlich gesprochen, der Couch entlang ziehen soll, einfach hingenommen!

\section{Der politische Raum - ein feindliches Territorium?}

DieAfrika-Forschungen der Parins und Morgenthalers, die eine Öffnung, auch eine Weite in die Diskussion brachten, liegen nun auch schon eine halbe Ewigkeit zurück. Und die später folgenden Arbeiten dieses Forschungstrios brechen, grob 
gesagt, in zwei Teile auseinander - in einen mehr historischen, narrativen und literarischen ${ }^{5}$ und in einen strenger am Subjekt orientierten, psychoanalytischen ${ }^{6}$, der den Spuren der inneren Repräsentanzen folgt, den Repräsentanzen des Sexuellen, der unbewussten Dynamik im Deutungsprozess und des Gesellschaftlichen. Dabei lässt sich zwar feststellen, dass die Parins - sowohl in den theoretischen Arbeiten als auch in den literarischen Werken - noch ständig nach den Schnittpunkten zwischen dem Entwicklungsverlauf unbewusster Kräfte und den ordnungsleitenden Mächten der gesamtgesellschaftlichen Zusammenhänge such(t)en, während bei Fritz Morgenthaler die gesellschaftliche Dimension dieses prekären Verhältnisses eine stärkere Wendung ins Apodiktische und damit auch Starre bekommt.

Allerdings sind die Unterschiede nicht immer gleich groß. Etwa seit Ende der 70er Jahre arbeiteten sowohl die Parins als auch Morgenthaler konsequent mit der - wie ich meine irreführenden - Dichotomie zwischen politischer Herrschaft und subjektiver Triebhaftigkeit, zwischen kultureller Ordnung und triebmotiviertem Unbehagen $^{7}$, um die zu beschreibenden analytischen Sachverhalte auch sozial und das heißt ab diesem Zeitpunkt auch meist in einem als feindlich empfundenen Territorium - verorten zu können. So ist die von Pierre Passett zitierte Metapher der Militärjunta, mit der Morgenthaler «das Stranguliert werden der Regungen des Primärprozesses durch die Ich-Organisation veranschaulichen» (1986: 168-69) will, keine zufällige sondern eine, die sich in eine ganze Assoziationskette einreiht, in der die Art der Organisation von gesellschaftlichen Institutionen in die Nähe der Zwänge gerät, die in der Rekrutenschule eingeübt werden:Von «falschem Bewusstsein» (Morgenthaler 1984: 95) ist die Rede, von «Zwängen» (1984: 97), «Belastungen» (1984:127) der «Drangsalierung» und «Disziplinierung» (1984: 143), ja sogar das Bild der «Schiessübungen» (ebenda) wird beigezogen. Und bei den Parins führt diese Dichotomisierung zu einem Rollenverständnis des Psychoanalytikers, das ihn in die Nähe eines Winkelrieds ${ }^{8}$ rückt: «Psychoanalytiker sind (...) in einer besonderen Lage. In ihrer täglichen Arbeit tiefemotional engagiert, stehen sie aufSeiten der unterdrückten Triebwünsche. (...) Da alle jene Kräfte, die zur Einengung und Verzerrung individuellen Seelenlebens geführt haben, Ausdruck undWirkung gesellschaftlicher Unterdrückung und Ursache des allgemeinen Unbehagens in der Kultur sind, üben Psychoanalytiker einen Berufaus, der sie ständig in die Lage unerbittlicher Kritiker ihrer Gesellschaft bringt (der sie ebenso wie die Analysanden angehören). Sie sind heimliche Subversive» (1985: 21)... womit die psychoanalytische Praxis, so wäre erstens anzufügen, zu einem gesellschaftlichen Ort verkommt, der sich, auf die Triebökonomie des Subjekts berufend, den gesellschaftlichen (Macht-)Verhältnissen entgegenstemmt; und zweitens und vor allem, dass im Bild des «heimlich Subversiven» 
auch das ideologische Gegenstück zum psychoanalytischen Funktionär enthalten ist! Dahinter verbergen sich die problematische Institutionsgeschichte des Psychoanalytischen Seminars sowie der bis zum heutigen Tag umstrittene Bedarf nach einer neuen ideologischen Basis.

Es würde sich deshalb lohnen, ein viel größeres Projekt in Angriff zu nehmen, nämlich eine eigentlich politische Publikationsgeschichte des PSZ zu schreiben, in der die verschiedenen Bücher, Artikel und Schriftstücke (nicht nur der Parins und Morgenthalers!) auch auf ihre impliziten Bilder des Gesellschaftlichen hin abgeklopft und mit der Geschichte einer Institution verklammert würden, deren Vertreter einmal glaubten, sich «desinstitutionalisieren» (Ideologie) zu können (und dabei den Formalisierungsgrad einer Institution permanent mit ihrer Existenz ${ }^{9}$ verwechselten). Auch scheint mir die ideologischeVerengung, von der ich berichte, in einem inneren Zusammenhang mit einer Institution zu stehen, deren Vertreter sich lieber als Teil einer «Bewegung» verstanden und darob übersahen, wie sie, weil sie das wirklich Unverständliche erklären mussten, in ein wachsendes Rechtfertigungsszenario hineinschlitterten, das in innere wie äußere Grabenkämpfe münden musste. Das von Johannes Cremerius gezeichnete Bild des Institutionellen kann deshalb auch als ein Versuch gelesen werden, die Geschichte der Institutionalisierung des PSZ mittels einer Karikierung der IPA-Organisation rückgängig machen bzw. vernebeln zu wollen. So schreibt der Autor im vom PSZ herausgegebenen Reader «Between the Devil and the Deep Blue Sea» (1987), dass die Institutionalisierung (wozu er den Marxismus und den Freudismus zählt) regelmäßig dazu führe, dass

, «der Institution (die) Essentialien der Theorie geopfert werden;

, der Institution Menschen geopfert (werden), die ihren Zielen im Wege stehen;

, die Institution ein Ausbildungssystem (schafft), das die Paradigmata des Gründers konservieren und die Adepten auf sie festlegen soll. Es dient mehr der Indoktrination als der Entfaltung wissenschaftlichen Denkens;

, (sie), damit sie dieses Ziel erreichen kann, (...) eine Machtstruktur braucht. Die schafft sie sich in einer hierarchischen Gliederung, in der es verschiedene Rangstufen der Macht gibt. Die Kontrolle der Macht wird den Mitgliedern entzogen;

, das kategoriale Instrument der Macht (...) das Zulassungsverfahren ist, das nur denen den Zugang ermöglicht, die sich unterwerfen und von denen gruppenkonformes Verhalten erwartet werden kann» (1987: 15).

Während also Cremerius Institutionen wie Personen behandelt, die aktiv etwas «schaffen», z. B. Ausbildungssysteme, und in institutionellen Regelungen 
nur dogmatische Strukturen erkennt, die zu nichts als Zumutungen, Zwängen und Beschränkungen führen, möchte ich deren konfigurative Funktion hervorheben: Überall, wo Menschen zusammenleben, entstehen Institutionen und Kulturen. Sie sind eine Art verstetigter Ausdruck bestehender Sozialbeziehungen, strukturieren die vielfach ineinander verschachteltenVerhaltenserwartungen undVerhaltensneigungen und führen zu Verhaltensregeln (Rechte und Pflichten), welche die Autonomie der Gesellschaftsmitglieder nicht nur beschränken, sondern gleichzeitig auch anerkennen. Die Schaffung von Institutionen ist eine der bedeutendsten menschlichen Kulturleistung, die es dem einzelnen ermöglicht, auf geschichtlich gewachsene Strukturen zurückzugreifen, die weit über die individuelle Lebensgeschichte hinausreichen und einen Fundus an Erinnerung bewahren, der für unser Überleben zentral ist.

Gerade wir Psychoanalytiker/innen müssten doch eigentlich um die konstitutive und konfigurative Bedeutung von Familien- undVerwandtschaftsverbänden wissen, die über die Produktion routinisierter Handlungsabläufe eine sehr wichtige und in mancher Hinsicht auch psychisch entlastende Funktion erfüllen. Seit vielen Jahren beschäftigt mich daher die Frage, wie es eigentlich dazu kommt, dass Personen, die täglich mittels der normativen Konstruktion des Settings jenen Prozess in Gang bringen, der Psychoanalyse erst ermöglicht, derart abwegige Überlegungen zur Bedeutung und Funktionsweise von Institutionen zum Ausgangspunkt der Corporate Identity ihres eigenen Seminars wählen. Denn ohne institutionelles Gedächtnis, ohne den koordinierten Austausch von Erfahrungen und Gedanken gäbe es auch gar keinen Fortschritt in der Psychoanalyse!

Die Absichtserklärung des PSZ, die seit den späten 70er Jahren bis zum heutigen Tag fortbesteht, behindert meines Erachtens eher die Entwicklung dieses Seminars, als dass sie der Profilierung dient, weil sie heute die Nichtformalisierung sich unter dem Teppich schon längst eingespielter (und ausgebreiteter) Machtregeln ${ }^{10}$ einem für alle transparenten Ordnungssystem weiterhin vorzuziehen scheint. Die so genannt institutionskritische Tendenz wendet sich gegen den institutionellen Hierarchisierungsgrad und bringt damit fortwährend zwei zentrale Fragen durcheinander, nämlich den Formalisierungsgrad einer Institution (ist das PSZ eine Institution oder eine Bewegung?) und die Frage, wer, welche Personen/Gruppen für sich das Normsetzungs- und vor allem das Normdurchsetzungsmonopol beanspruchen, etwa mittels der Etablierung neuer Argumentationsketten und Diskursordnungen. Wenn also transparente Ordnungssysteme - so will es die PSZ-Ideologie - nichts als Blockaden schaffen ${ }^{11}$, fragt sich auch: Für wen? Und weiter: Weshalb schon längst eingespielte Machtregeln, wenn sie doch nur Blockaden schaffen, von einer 
auf den gesellschaftlichen Wandel abzielenden psychoanalytischen Institution in den politischen Papieren so selten thematisiert werden ${ }^{12}$ ? Anders herum: Geht es hier um eine Diskursordnung der Konfliktverleugnung, indem die ständig von neuem zelebrierte Erinnerung an die Abspaltungsgeschichte («Waserstrasse», «Schlüsselübergabe», «SGP», «IPV» und anderem mehr) unter anderem dazu dient, auf das unter dem Teppich Drängende gerade nicht zu sprechen zu kommen? Inwieweit steht das unter dem Teppich Drängende ${ }^{13}$ in einem inneren Zusammenhang mit dem äußerst mangelhaft geführten Diskurs zum Verhältnis Psychoanalyse-Politik/ Gesellschaftskritik ${ }^{14}$ ?

Hier möchte ich einhaken und auf einige konzeptionelle Probleme hinweisen, die in erster Linie den politischen Diskurs am PSZ verstopft haben. Ich halte mich dabei nicht an geschichtliche Abfolgen, sondern folge den Spuren meiner Assoziationen, die mich auf verschiedene Ideen brachten.

\section{$3 \quad$ Begann alles hinter der Couch?}

Vor nunmehr zwanzig Jahren, in der «Journal»-Sondernummer «Institutionalisierung - Desinstitutionalisierung» hat Ralph Binswanger (1987) in großen Linien die Voraussetzungen skizziert, die zur unseligmachenden Forderung führten, es solle eine Teilung der psychoanalytischen Berufsidentität eingeführt werden, die sich, bildlich gesprochen, der Couch entlang zieht: Hinter der Couch sollte subtil, professionell und analytisch gearbeitet werden; vor und außerhalb der Couch - also im politischen Feld - so interventionistisch, wie es unsere Fähigkeiten gestatteten. Spuren dieser Metapher finden sich in Berthold Rothschilds Bild der «fünf Finger der linken Hand» (1988: 8-10) wieder, nämlich im vierten, dem Ehefinger und im ach so kleinen fünften ${ }^{15}$. Er steht für die säkulare Richtung und wendet sich mitunter gegen die «tendenzielle Selbstüberschätzung der Psychoanalytiker als Kultur- und Geistesträger par excellence» und gegen «die ubiquitäre Tendenz, alles und jedes - fernab von der Couch - zu deuten und unter die Fittiche des psychoanalytischen Verstehens zu nehmen.» Besonders beschäftigt ihn aber den Ehefinger, dem er die "postmoderne» Strömung zuordnet: «Sie propagiert die Psychoanalyse als radikales Denkgebäude, will sie wohl in der Nachfolge der strukturalistischen und hermeneutischen «Staatsstreiche〉, zur reinen Geisteswissenschaft deklarieren. Sie nimmt Äquidistanz zu allen Formen psychoanalytischen Tuns, sucht ihr Engagement in der Radikalität des psychoanalytischen Denkens überhaupt, denunziert politisches Engagement des Psychoanalytikers (nicht als Privatperson, sondern in seiner psychoanalytischen Funktion) als «Verrat` an der psychoanalytischen Radikalität (...)» (1988: 9). Institutionsgeschichtlich betrachtet möchte ich diese 
von Rothschild als «säkulare» bzw. als «postmoderne Strömung» bezeichneten Tendenzen neu einordnen. Während die säkulare Strömung für eine entschiedene Abkehr vom missionarischen Eifer psychoanalytischen Deutens außerhalb von Couch und Chaiselongue steht, kehrt das Verschleiernde, Verdunkelnde in Form der «Postmodernität» wieder. Denn «postmodern» ist ja bekanntlich alles, was nach der üblichen Zeitrechnung folgt, sich zeitlich also nicht recht einordnen lässt und sich auch in diesem Fall auf etwas anderes bezieht: auf die (institutions-)geschichtlich geprägte Reaktion auf einen diskursiven Machtverlust im öffentlichen Raum, weshalb auch von «Staatsstreich» ${ }^{16}$ die Rede ist. In Wirklichkeit weist diese als "postmoderne Strömung» verkleidete Tendenz sowohl rückwärts, nämlich in Richtung der bis heute noch aktuellen Abspaltungsgeschichte ${ }^{17}$ und in Richtung einer letztlich reaktiven,Radikalisierung des psychoanalytischen Denkens als auch nach innen, nämlich in Richtung der in Frage gestellten politischen bzw. offentlichen Identität der/des Psychoanalytikers/in (Legitimationsproblematik). Ihr Produkt ist die von mir beklagte Selbstvergewisserungstendenz im öffentlichen Raum. «Säkulare» und "postmoderne Strömung» müssen vor dem Hintergrund des Binswangerschen Couch-Konzepts diskutiert werden. Sie signalisieren einen ideologischen Wandel, der bis heute nachwirkt. Doch schön der Reihe nach:

Schon der Titel des Artikels von Ralph Binswanger, «Die Währung in der Psychoanalyse und die Währung in der Politik», steht in einem eigenartigen Missverhältnis zu dessen Inhalt. Ließe die gewählte Überschrift erwarten, dass Binswanger sich zum prekärenVerhältnis Psychoanalyse-Politikäußerte ${ }^{18}$, so bemerkt der Leser sogleich, dass dem Autor das eigene Hemd viel näher liegt, als der fremde Rock. Binswanger interessiert sich für eine ganz andere Fragestellung, nämlich «was ein «linker Psychoanalytiker (sei), was das «Linkssein in der Psychoanalyse (ausmache)» und «was das «Linkssein einer psychoanalytischen Gruppierungoder Institution (sei)?» (1987:12) Mit anderenWorten: Ihm geht es um ein Identitätsproblem, das den erwähnten Bedarf einer ideologischen Basis signalisiert (>Institutionsgeschichte):

«Es hat sich gezeigt - z.B. durch die friedliche Koexistenz von Waser- und Quellenstrasse (...) dass dieser Kampf keine Identität als linke Psychoanalytiker mehr stiften kann. Sie muss in dem gesucht werden, was wir inhaltlich vertreten und was wir konkret tun. Dabei geht es um zwei Bereiche möglicher Aktivität: innerhalb der psychoanalytischen Tätigkeit mit Klienten einerseits, außerhalb derselben, im sozialen resp. politischen Bereich andererseits. Im zweiten Bereich stellt sich die Frage: Können wir spezifisch als «linke〉 Psychoanalytiker politisch wirksam werden? Gibt es auf dieser Ebene den 'Partisanenkampf mit anderen Mitteln〉, wie Parin das ausdrückte?» (ebenda, Hervorhebung im Text). Natürlich gibt es das 
- aber dazu später. Und auf der nächstfolgenden Seite: «Innerhalb meiner Arbeit als Psychoanalytiker muss ich das «Linkssein also immer in die psychoanalytische Währung übersetzen und diese «vergolden`. Dies bedeutet, den radikalen Kern des Freud'schen Ansatzes freizulegen und wenn möglich zu vertiefen. ${ }^{19}$ Es geht darum, sich die zentralen psychoanalytischen Konzepte ${ }^{20}$ (das Unbewusste, das Sexuelle, den Konflikt und damit die Konflikthaftigkeit beider Partner im Zweipersonenfeld etc.) nicht revisionistisch vernebeln zu lassen» (1987: 13). Somit gründet Binswangers Rede auf einer Verteidigungsposition. Sie wendet sich gegen die neuen Moden und ist in ihrer Quintessenz rückwärtsgewandt («Back to the roots!» ${ }^{21}$ ). Sie klingt wie das Parteiprogramm einer alten Psychoanalytiker-Zunft, die qua einer linken Vernebelungstaktikihre konservative Stossrichtung verdunkelt und im öffentlichen Raum für den Stellenwert der eigenen Sache kämpft. Damit steht Binswangers Plädoyer zwar in einem inneren Zusammenhang mit der heute betriebenen Standespolitik. Denn auch sie beschränkt das Politische auf die Vertretung der eigenen, im standespolitischen Fall höchst pekuniären Interessen.

Aber: Damals, als es zum Aufbruch gegen das Psychoanalyse-Verständnis der IPA kam und an derVia Trionfale 200 in Roma ein Gegenkongress organisiert wurde, ging es noch nicht um bloße Interessenpolitik. Auch war damals der Kampf, das politische Engagement, ein Mittel, um eine institutionelleVeränderung zu erreichen - und (noch) nicht identitätsstiftender Teil «an sich». Es ging doch darum, die Art, wie Psychoanalyse in den verschiedenen Gesellschaften verwaltet wurde, in Frage zu stellen. Hintergrund dieser Infragestellung war ein neu erwachtes politisches Bewusstsein, nämlich die Demokratisierung weiterzutreiben und institutionalisierte Machtbildungsprozesse an sich zu hinterfragen, ${ }^{22}$ weil man/frau sich davon größere Mündigkeit, mehr Autonomie, Selbstverwirklichung, eine erfolgreichere, der Aufklärung stärker verpflichtete Psychoanalyse und damit vielleicht sogar ein «schöneres Leben» versprach. Eine Durchsicht der Artikel der erwähnten «Journal»Sondernummer von 1987 zeigt indessen, dass eine Debatte über den Wert der politischen Stossrichtung bereits damals nicht mehr aktuell war. ${ }^{23}$ Geblieben ist stattdessen ein Grad an gesellschaftspolitischer Selbstbezogenheit, der eigentlich hätte stutzig machen müssen. Hätte ...

\section{Währungsprobleme}

Bezeichnend für diesen, letztlich legitimatorisch begründeten Rückfall ist die Stossrichtung von Binswangers «Zweiwährungstheorie»: «Wenn ich in einem psychoanalytischen Prozess eine Hypothese bilde, wenn ich aufgrund dieser Hypothese zum Beispiel eine Übertragungsdeutung gebe, so wird sich diese verifizieren oder 
falsifizieren. Die Theorie der Technik nennt verbindliche handwerkliche Regeln dafür. Zur Verifikation einer Übertragungsdeutung gehören die Entspannung der Beziehung, eine aufdie Deutung folgende, für beide Partner evident werdende Antwort des Unbewussten und das Aufkommen lebendiger Einfälle aus dem jetzigen und vergangenen Leben des Analysanden, welche die gedeutete Übertragungssituation als Wiederholung früherer Konfliktlösungsmuster erkennbar machen. Die psychoanalytische Deutung ist gleichsam die Münze, die ich hergebe, um etwas Bestimmtes dafür zu bekommen. Im Reich der Psychoanalyse gibt es eine spezifische Währung, die (...) gehandelt wird. Gehe ich nun mit dieser Währung ins Reich der Politik, ist es völlig ungewiss, ob sie dort überhaupt eingetauscht wird. Vielleicht bekomme ich schon irgendetwas (...), vielleicht aber nur eins aufs Dach. Mit anderen Worten: Wie verifiziere ich außerhalb der psychoanalytischen Situation meine Konzepte? Es gibt meines Wissens keine Möglichkeit. Es bleibt dem Zufall, vielleicht der ‘Intuition〉 überlassen, ob psychoanalytische Aufklärung in politischen Situationen mehr istals eine schöngeistige Übung. Das ist wahrscheinlich noch zu wohlwollend formuliert, und wir können noch einen Schritt weiter gehen: Politische Interventionen, die mit psychoanalytischen Begriffen aufklärend wirken wollen, sind grundsätzlich nichts anderes als «wilde` Deutungen, also Deutungen außerhalb eines Auftrags, außerhalb einer psychoanalytischen Situation, was erfahrungsgemäßzu nichts anderem führt als zum Aufbau unangehbarer Widerstände» (1987:13). Hier begegnen wir wiederum diesem eigentümlichen Phänomen, nämlich dass das «Reich der Politik» zum feindlichen Territorium erklärt wird ... «(gehe) ich (...) ins Reich der Politik, (...) bekomme ich (...), vielleicht (...) nur eins aufs Dach» (ebenda). ${ }^{24}$ Sodann greift Binswanger ein Kernproblem auf, das sich an der Grenze zwischen dem «Reich der Psychoanalyse» und dem «Reich der Politik» stellt, nämlich wie die am Subjekt und am psychoanalytischen Setting orientierte Arbeitsweise in die Arbeit mit politischen Zusammenhängen eingefügt werden kann. Doch wird gar keine Suche nach Antworten in Gang gesetzt, die diese Grenze ausreizte und das Denken weitertriebe, sondern es wird zum Mittel der Entwertungen gegriffen: Diejenigen, die es trotz der skizzierten Schwierigkeiten dennoch versuchen wollen, geraten mit dem Verweis auf «Zufall», «Intuition» und «wilde Deutungen» (1987: 12) in den Geruch des Unanalytischen, des Unprofessionellen und des «Verrats» an der gemeinsamen Sache. Diskursive Rechtfertigungsszenarien und sinnlose Grabenkämpfe, die bis heute fortwirken, sind die Folge.

«Politisch kann (der Aufbau unangehbarerWiderstände/MW) nur zu negativen Reaktionen bei denen führen, die man aufklären und beeinflussen will (...).Zusätzlich führteine solche politische Praxis zur Desavouierung der Psychoanalyse als Ganzes. Sie 
verstellt den Blick (...) des so agierenden Psychoanalytikers für das, was politisch real stattfindet. Sie hindert ihn, politisch wirklich aktiv zu werden»(ebenda)....Natürlich, recht hat er, unangehbare Widerstände sollte man/frau nicht produzieren, weder im psychoanalytischen Setting noch außerhalb, das ist ein Kunstfehler - aber:

, Erstens:Man/fraugeht-bildlich gesprochen-jaauchnichtmitSchweizerfranken nach Italien, um sich dort etwas Schönes zu kaufen. Um den Kauf zu tätigen, wird irgendwann die Grenze überschritten und es wird dieWährung gewechselt, sei es in einerWechselstube oder einer Bank. Wer trotz solcher Einrichtungen an seinen schweizerischen Noten und seinem Kleingeld festhält, produziert unter Umständen nichts als «unangehbare Widerstände» und macht sich das Leben selber schwer. Daraus zu schließen, man/frau solle auf den Kauf verzichten und besser in der Schweiz bleiben, weil es sonst nur zu «wilden» Transaktionen komme, ist weder zwingend noch überzeugend.

, Zweitens ist mir noch heute unverständlich, weshalb Psychoanalytiker, die sich ins politische Feld begeben, dort à tout prix mit psychoanalytischen Begriffen aufklärend wirken wollen. Einmal abgesehen davon, dass diese Absicht unanalytisch ist, so ist sie auch nicht zwingend. Auf dieser Basis ${ }^{25}$ wird jedenfalls kein Dialog in Gang gesetzt und eine Institution, die Fragen zum Verhältnis von Psychoanalyse und Politik bzw. Psychoanalyse und Gesellschaft zu den Bausteinen ihrer Selbstdefinition erklärt hat (Viersäulenmodell), wäre gut beraten, gerade diesen Dialog zu suchen. Das daran gebundene Sendungsbewusstsein weist deshalb auch nicht vorwärts, in Richtung einer Öffnung, wie sie noch in den Afrika-Studien von Parin/Morgenthaler aufscheint, sondern rückwärts, nämlich in Richtung einer irgendwie gearteten politischen Psychoanalytikeridentität (Selbstvergewisserungstendenz). Weshalb sollte denn das politische Feld nur als erweiterter Aktionsraum der eigenen Mission benutzt werden? Und weshalb soll die ganze Spannkraft, die vom fremden Erkenntnisbereich ausginge, in das eigene Weltbild eingegossen und somit «gefressen» werden?

InderanalytischenSituation arbeitet deroder diepraktizierendePsychoanalytikerIn zusammen mit seinen oder ihren Analysand/inn/en innerhalb eines klar umrissenen und auf die analytische Beziehung abgestimmten normativen Settings an deren Lebensgeschichten. Über den Dreischritt von Wiederholen, Deuten, Durcharbeiten werden Erlebnisse mit Erinnerungsspuren verknüpft, um Schritt für Schritt ein komplexes Netz biographischer Erinnerungen und Erfahrungen zu heben und bewusst zu machen. Ginge Binswanger mit dem Wissen um die konstitutive Bedingung des psychoanalytischen Prozesses in das Feld der Politik und würde dort also in Analogie 
zur psychoanalytischen Anlage ein Setting entwickeln, das es ihm erlaubt, über die Aufarbeitung der unmittelbaren Beziehungen hinaus vor allem kulturgeschichtliches Material bereitzustellen, um darin konsistent zu deuten ${ }^{26}$, käme es wohl nicht zu solchen Konfusionen. Sie entstehen, weil der Autor dort von etwas anderem berichtet, nämlich von einem missratenen Sendungseinsatz. «So what?»

Um den Dialog an der Grenze zwischen Psychoanalyse und Politik bzw. Psychoanalyse und Gesellschaft führen zu können, ist es sehr wichtig, dass die entsprechenden Inhalte nicht politisiert und damit ideologisch aufgeladen werden. Agieren ist nicht nur im Rahmen psychoanalytischer Prozesse, sondern in jedem Fall daneben. Als äquivalentes Motto zum psychoanalytischen Prozess könnte zum Beispiel formuliert werden: «Politische Prozesse bewusst machen - nicht (Partei-) Politik machen!» - will heißen: sich nicht in das politische Tagesgeschäft einmischen, sondern die gesellschaftlichen Strukturkonflikte in ihren Verhängtheiten mit den inneren Widersprüchen im Subjekt auf die Agenda setzen ${ }^{27}$. Die Frage des «Links-Seins» ist in solchen Zusammenhängen nicht nur «suboptimal», sondern kontraproduktiv. In Tat und Wahrheit ist es nämlich nicht die psychoanalytische Position, sondern um die politische Ideologie (und die daran gebundene Institutionsgeschichte des PSZ), die die Glaubwürdigkeit der vertretenen Inhalte im öffentlichen Raum permanent untergräbt, weil sie insinuiert, der vertretene Standpunkt sei nicht empirisch sondern ideologisch bzw. institutionsgeschichtlich determiniert. Eine solche Tendenz verstellt die Denkräume und verkehrt den Dialog, auf den das Seminar so dringend angewiesen wäre, in einen äußerst unersprießlichen Schlagabtausch. Denn psychoanalytisches Denken ist weder «links» noch «rechts» sondern anders (als das im gesellschaftswissenschaftlichen Diskurs dominierende kognitive, strategische und normative Denken).

\section{$5 \quad$ Die Selbstvergewisserungstendenz}

Die von mir monierte Selbstvergewisserungstendenz möchte ich nun anhand des von Pierre Passett, Olaf Knellessen und Peter Schneider verfassten Artikels «Parin, Morgenthaler und die Politik» (1993) veranschaulichen. Ihr Untertitel verspricht «eine Skizze zur Psychoanalyse des Politischen und Gesellschaftlichen (...)» (1993: 143/Hervorhebung MW), doch folgt die Argumentationslinie nur der psychoanalytischen Logik. Im Bemühen, à tout prix radikal psychoanalytisch sein zu wollen, steht nicht eine Auseinandersetzung mit den politischen und gesellschaftlichen Inhalten der Autoren im Mittelpunkt, die sie mit psychoanalytischen Mitteln zu erschließen versuchten, sondern «bloß» die Frage, was die psychoanalytische Qualität 
dieser Studien ausmache, ob Parins und Morgenthalers Sicht des Gesellschaftlichen psychoanalytisch vertretbar sei:

«(...) Die Psychoanalyse zeichnet sich in Parins Deutung durch ein umgrenztes Gegenstandsfeld aus, wobei die auf diesem Gebiet gewonnen Forschungsergebnisse in ein umfassenderes interdisziplinär humanwissenschaftliches Theoretisieren eingefügt werden sollen (1993: 154). (...) In dieser Beschreibung (d.i. die Sicht Parins) scheinen sich Subjekt und Gesellschaft (dessen soziale Realität) in einer bemerkenswert unabhängigen Parallelität zueinander zu entwickeln. Zwar kommt es zu Kollusionen und Konflikten, doch ist nichts davon zu spüren, in welch prekärer Verhängtheit sich die beiden befinden, jene Verhängtheit, die etwas damit zu tun hat, dass sich Gesellschaft und Kultur einer ‘seltsamen〉 Perversion ${ }^{28}$ der Sexualität beim Menschen verdanken»(1993: 158). Und dann: «Im strengen Sinne psychoanalytisch kann Gesellschaft nur verstanden werden als das Insgesamt jener Beziehungen zwischen den Menschen, die sich der problematischen Anlehnung des Sexualtriebes an die Selbsterhaltungstriebe verdanken. Und alle diese Beziehungen sind letztlich gespeist von einem Begehren gegenüber Anderen, in deren Spiegelung wir uns zugleich er- und verkennen und wir wiederum im Spiegel unseres Selbst erkennen können und damit notwendig eben auch verkennen müssen. Psychisch und Physisch haben wir keine Chance (...) ohne diese Anderen und ihre Zuwendung zu überleben und können doch zugleich auch nichts dagegen tun, dass unsere Hinwendung zu diesen Anderen immer eine Grenzüberschreitung und damit Gewaltausübung impliziert, so dass der Konflikt mit diesen Anderen nicht Resultat eines (zufälligen) Versagens ist, sondern notwendige Reproduktion der eigenen zerrissenen Verfassung»(ebenda). «(...) Allerdings glauben wir, dass dieser Zusammenhang (zwischen individueller und gesellschaftlicher Konflikthaftigkeit/MW) gerade nicht in erster Linie so zu sehen ist, wie Parin ihn darstellt, dass sich die gesellschaftlichen Konflikte in den individualpsychischen abbilden und dass sie diese wesentlich bedingen, sondern so, dass die gesellschaftlichen Konflikte in einer Weise, die erst noch zu erfinden und adäquat zu beschreiben ist, als Inszenierungen, Austragungen jener Widersprüche im Subjekt zu begreifen sind, die diesem Subjekt nicht nur notwendigerweise zugehören, sondern die es eben erst konstituieren, und damit auch seine notwendige Gesellschaftlichkeit»(1993: 159).

MitanderenWorten:Eswird nur die seelisch dispositive Seite des Gesellschaftlichen beschrieben, die Geschichte des Wunsches nur im individuellen Subjekt ${ }^{29}$ verankert (vgl. das «Begehren des Anderen»), die Grenzüberschreitung des Subjekts nur mit Gewaltausübung in Verbindung gebracht und einem eigenartigen (und institutionsgeschichtlich betrachtet letztlich reaktiven) psychoanalytischen Monismus das 
Wort geredet und nicht, wie noch bei Parin/Morgenthaler, nach den Schnittpunkten zwischen den beiden grundverschiedenen Logiken, dem psychoanalytischen und dem gesellschaftswissenschaftlichen Diskurs des Politischen gesucht. Wird der gesellschaftliche Raum in marxistischen Theorien auf die Produktivkräfte der Ökonomie reduziert, so verkommt er hier zu einem erweiterten Raum eines individuellen Subjekts, das sich anhand von Selbst(be-)spiegelungen seinen eigenen Reim über sich und dieWelt macht und aufgrund seiner eigenen Konflikthaftigkeit so oder anders mit derWelt interagiert. Diese «Gesellschaftsdefinition» (ich setze sie in Anführungsstriche, weil sie keine ist, da sie auf das überindividuelle komplexe Beziehungsnetz und dessen Geschichte gar nicht eingeht) liest sich für mich wie der Nachklang einer Kohutschen Gesellschaftstheorie, die es freilich nie gegeben hat. Gleichzeitig fallen mir dabei Alexis de Toqcuevilles Gedanken zum Übergang von der Aristokratie in die Demokratie ein: «L'aristocratie avait fait de tous les citoyens une longue chaîne qui remontait du paysan au roi; la démocratie brise la chaîne et met chaque anneau à part. (...) Ainsi, non seulement la démocratie fait oublier à chaque homme ses aïeux, mais elle lui cache ses descendants et le sépare de ses contemporains; elle le ramène sans cesse vers lui seul et menace de le renfermer enfin tout entier dans la solitude de son propre coeur» (1968 : 244). Die Idee, die subjektive Konflikthaftigkeit mit den gesellschaftlichen Konfliktlinien zu verklammern, setzt nicht nur eine Spiegelungshypothese ${ }^{30}$ sondern auch ein Neuverständnis des Aggressionstriebes (warum soll Aggression nur destruktiv sein? fragt Emilio Modena mit Recht ${ }^{31}$ ) und vor allem die Aufarbeitung politischer und kultureller Geschichte und die Geschichte von Rollenintegraten und Statusbeziehungen voraus sowie eine Analyse des komplexen Interaktionsfeldes zwischen Subjekt, Familie und Kultur (vgl. Erdheim 1982). Während Parin/Morgenthaler anhand ihrer Afrika-Studien sich auf dieses Wagnis eingelassen haben und anhand konkreter gesellschaftlicher Zusammenhänge den Spuren der Schnittpunkte zwischen «innerer», subjektiver und «äußerer», gesellschaftlicher Konflikthaftigkeit gefolgt sind, verkommt hier wie auch in einer späteren Arbeit der selben Autoren ${ }^{32}$ das Denken über gesellschaftliche Zusammenhänge zu einem losen Philosophieren über «das Gesellschaftliche» - ohne jeden konkreten Realbezug! Rein assoziativ lassen sich noch viele Verknüpfungen herstellen und rein theoretisch mag noch vieles einleuchten, ich möchte aber doch erinnern, dass die Aneignung psychoanalytisch relevanten Wissens genauso wenig wie die Aneignung gesellschaftswissenschaftlich relevanten Wissens eine ausschließliche Angelegenheit der Bibliothek ist. So wenig, wie es genügt, sich in andere Menschen bloß «hineinzuversetzen», genügt es, sich gesellschaftspolitische Zusammenhänge bloß vorzustellen, um ein Konzept der interaktiven Dynamik 
unbewusster Konflikte zu entwickeln. Und die ganz andere Frage, nämlich ob die von Parin/Morgenthaler dokumentierte Sicht von Psychoanalyse des Politischen im gesellschaftswissenschaftlichen Diskurs vertretbar sei, welche politischen und welche sozialwissenschaftlichen Implikationen, welche Grenzen und welche Potentiale sie enthalte, ${ }^{33}$ bleibt ganz ausgespart. Dabei wäre es doch gerade diese zweite Frage, die die Psychoanalyse aus dem klinischen Raum hinausführte und ihr mit Recht einen sicheren Platz innerhalb der gesellschaftswissenschaftlichen Debatten zuwiese.

\section{Epilog}

Man mag über den Wert meiner Kritik geteilter Meinung sein, etwa hervorheben, dass die politische Dimension innerhalb des PSZ noch nie eine große Rolle gespielt habe, weil sie von der breiten Basis gar nicht mitgetragen wurde, sondern immer die Angelegenheit ein paar Weniger war, die nun auch ins Alter kommen; oder dass schon immer die Frage, wie man/frau die eigenen Brötchen ins Trockene bringe, das Denken über Politik geprägt habe. Das mag alles seine Berechtigung haben, aber es ist ein Irrtum anzunehmen, dass diejenigen, die gar nie in den hier skizzierten Diskurs eingegriffen haben, von diesem unberührt blieben, noch ist es so, dass nur pekuniäre Fragen das Denken über Politik prägen. Auch Politik wirkt, ob einem das jetzt passt oder nicht und auch die Frage, wie das PSZ von außen wahrgenommen wird, ist heutzutage alles andere als belanglos. Eine Institution aber, die seit Jahrzehnten das Viersäulenmodell auf ihre Fahnen geschrieben und zum Beispiel Geld für die bewegten Jugendlichen oder den Befreiungskampf in Nicaragua gesprochen hat, aber den entsprechenden gesellschaftswissenschaftlichen Anspruch bis zum heutigen Tag nicht eingelöst hat, schlittert mit Sicherheit in ein Legitimationsproblem hinein, das den öffentlichen Auftritt nachhaltig belastet. Denn wie kann - um nur einige der Fragen zu nennen - das PSZ seine für die eigene Identität konstitutive Abspaltungsgeschichte produktiv weiterführen, wenn sich die Größe des PSZ weniger auf eine inhaltliche Abgrenzung zu einem rein klinischen Psychoanalyseverständnis als vielmehr auf das (qualitativ freilich hoch stehende) Dumpingpreisangebot zurückführen lässt, mit dem eine Psychoanalyseausbildung am PSZ zu haben ist? Wie soll das PSZ im öffentlichen Raum in Zukunft an inhaltlichem Profil gewinnen, wenn sich bei nüchterner Betrachtung zeigt, dass insbesondere die vierte Säule, die das PSZ von den übrigen Instituten am wohl deutlichsten unterscheidet, einem wachsenden psychoanalytischen Monismus zum Opfer fällt, der bei anderen Instituten schon immer «Mode» war? 
Gerade wir Psychoanalytiker/innen müssten doch wissen, dass es nicht einerlei ist, wie institutioneller Anspruch und Wirklichkeit zueinander stehen ${ }^{34}$ und dass derartige Diskrepanzen mit allen Diskursteilnehmern auch etwas machen. Der in diesem Zusammenhang nicht selten zu hörende, äußerst formalistische (und legalistische) Einwand, nämlich dass das PSZ als «einfache Gesellschaft» (Norm) gar nie einen solchen Homogenitätsgrad (Verhalten) erreicht habe, als dass man/frau das PSZ als Ganzes - eben als eine institutionelle Körperschaft ansprechen könne, gehört zur skizzierten Diskursordnung der Konfliktverleugung. Er ist äußerst fadenscheinig, dient bloß der Vernebelung der hier angesprochenen Sachverhalte und ist - gerade aus einer gesellschaftskritischen Sicht - ganz entschieden zurückzuweisen. Denn wie die Mitglieder eines Verbandes über ihre Beziehungen denken und wie sie sich diese Beziehungen zurechtlegen (Ideologie), welche Bereiche sie formalisieren möchten und welche nicht usw. und wie solche Verhaltensweisen, die natürlich einer inneren Logik folgen, von außen zu bewerten sind, sind zwei ganz verschiedene paar Stiefel. Eine Institution aber, die über ihre Selbstbeweihräucherungstendenzen hinauskommen will, wäre gut beraten, sich auch dem gesellschaftswissenschaftlichen «Courant normal» über die Bildung institutioneller Beziehungen zu stellen, statt - wie etwa die SVP den Sonderfall Schweiz-immerzu nur den eigenen, besonderen Status herunterbeten zu wollen. Voraussetzung dazu ist die institutionelle Etablierung eines qualifizierten, gesellschaftswissenschaftlichen Lehrangebots.

\section{Literatur}

Cremerius, J. (1987): Unterdrückung von Wahrheit, persönlicher Freiheit und wissenschaftlichem Denken in der psychoanalytischen Bewegung. In: Between the Devil and the Deep Blue Sea, Psychoanalytisches Seminar Zürich (Hg.), Freiburg i. Br.: Kore Verlag: 15-34.

Bazzi, D. (1987): Die Institutionsanalyse als theoretisches Werkzug für die Utopie der autonomen Institution, Journal, Psychoanalytisches Seminar Zürich, Sondernummer: $4-6$.

Bendkower, J. (1987): ... und nun entscheide dich, welchem Selbstmissverständnis der Psychoanalyse du folgen willst ..., Journal, Psychoanalytisches Seminar Zürich, Sondernummer: 17-25.

Bendkower, J., Merzbacher, K., Kappeler, Th. und Strassberg, D. (1980): Von den Schwierigkeiten, die Regressionen der Couch in einer demokratischen CouchInstitution zu überwinden - oder: Die Tellstrasse aus der Froschperspektive, in: Journal, Psychoanalytisches Seminar Zürich, Nr. 1: 2-6. 
Binswanger, R. (1987): Die Währung in der Psychoanalyse und die Währung in der Politik, Journal, Psychoanalytisches Seminar Zürich, Sondernummer: 12-13.

Bourdieu, P. (1979): Entwurf einer Theorie der Praxis. Frankfurt: Suhrkamp TB.

Busch, H. J. (2000): Die Aktualität psychoanalytischer Sozialpsychologie, Journal, Psychoanalytisches Seminar Zürich, Nr. 39: 48-62.

Devereux, G. (1978): Ethnopsychoanalyse, Frankfurt am Main: Suhrkamp, Weißes Programm

Dreyfuss, M. (1987): Eine kritische These über die Folgen unserer Art, Psychoanalyse zu vermitteln, Journal, Psychoanalytisches Seminar Zürich, Sondernummer, S.31-33

Erdheim, M. (1982): Die gesellschaftliche Produktion von Unbewusstheit. Eine Einführung in den ethnopsychoanalytischen Prozess, Frankfurt am Main: Suhrkamp.

Freud, S. (1982): Totem und Tabu. Einige Übereinstimmungen im Sexualleben der Wilden und der Neurotiker, in von Uexküll, Thure und Ilse Grubrich-Simitis (Hg.), Fragen der Gesellschaft. Ursprünge der Religion: 287-444 Frankfurt am Main: Fischer Taschenbuch Wissenschaft. Erstpublikation 1912-1913.

Giddens, A. (1984): Die Konstitution der Gesellschaft. Grundzüge einer Theorie der Strukturierung. Mit einer Einführung von Hans Joas, Frankfurt / New York: Campus Verlag.

Grosz, P. (1987): Identität und Veränderung, Journal, Psychoanalytisches Seminar Zürich, Sondernummer: 30-31.

Modena, E. (2000): Selbstverwaltete Psychoanalyse-Zürich zum Beispiel, Journal, Psychoanalytisches Seminar Zürich, Nr. 39: 22-33.

Modena, E. (2000b): Aggredo und Libido. Zur Entmythologisierung der Freudschen Triebtheorie. Psychosozial, Heft 84: 15-55.

Morgenthaler, F. (1978): Technik. Zur Dialektik der psychoanalytischen Praxis, Frankfurt am Main: Syndikat Verlag.

Morgenthaler, F. (1984): Homosexualität, Heterosexualität und Perversion, Frankfurt am Main: Qumran.

Morgenthaler, F. (1986): Der Traum. Fragmente zur Theorie und Technik der Traumdeutung, Frankfurt am Main: Qumran.

Müller, H.-P. (1987): Über die Schwierigkeiten, ein nicht-hierarchisiertes Seminar zu sein, in: Journal, Psychoanalytisches Seminar Zürich, Sondernummer: 14-17. 
Knellessen, O., Passett, P. und Schneider, P. (1993): Parin, Morgenthaler und die Politik. Eine Skizze zur Psychoanalyse des Politischen und Gesellschaftlichen und ihrerTradition am Psychoanalytischen Seminar Zürich, in: Luzifer-Amor, Zeitschrift zur Geschichte der Psychoanalyse, 6. Jahrgang, 12: 143-165.

Knellessen, O., Passett, P. und Schneider, P. (1995): Die Verflüchtigung und Wiedergewinnung des Politischen, Journal, Psychoanalytisches Seminar Zürich, Nr. 30: 22-47.

König, R. (1979): Soziologie. Das Fischer Lexikon, Frankfurt am Main: Fischer Taschenbuchverlag, Erstausgabe 1958.

Kurz, T. (1993): Aufstieg und Abfall des Psychoanalytischen Seminars Zürich von der Schweizerischen Gesellschaft für Psychoanalyse, Luzifer-Amor, 6. Jahrgang, 12: 7-54.

Parin, P. (1978): Der Widerspruch im Subjekt. Ethnopsychoanalytische Studien, Frankfurt am Main: Syndikat.

Parin, P. (1982): Untrügliche Zeichen von Veränderung. Jahre in Slowenien, Frankfurt am Main: Fischer Taschenbuchverlag.

Parin, P. (1985): Zu viele Teufel im Land, Frankfurt am Main: Syndikat.

Parin, P. (1990): Noch ein Leben. Eine Erzählung. Zwei Versuche, Freiburg im Breisgau: Kore.

Parin,P.(1995):EineSonnenuhrfürbeideHemisphären. UndandereErzählungen,Hamburg: Europäische Verlagsanstalt.

Parin, P. und Parin-Matthèy, G. (1986): Subjekt imWiderspruch, Frankfurt am Main: Syndikat Verlag.

Parin, P., Morgenthaler, F. und Parin-Matthèy, G. (1967): DieWeißen denken zuviel. Psychoanalytische Untersuchungen in Westafrika, München: KindlerVerlag, Reihe Geist und Psyche.

Parin, P., Morgenthaler, F. und Parin-Matthèy, G. (1978): Fürchte deinen Nächsten wie dich selbst. Psychoanalyse und Gesellschaft am Modell der Agni in Westafrika, Frankfurt am Main: Suhrkamp Taschenbuch Wissenschaft.

Passett, P. (1986): Die Aufhebung des Widerspruchs in der Bewegung. Eine AuseinandersetzungmitFritz Morgenthalers metapsychologischen Reflexionen zur Sexualität, in: Psychoanalytisches Seminar Zürich (Hrsg.), Sexualität. Frankfurt am Main: Syndikat/EVA: 157-203.

Popitz, H. (1968): Prozesse der Machtbildung, in: Reihe Recht und Staat in Geschichte und Gegenwart, Heft 362/363, Tübingen: J.C.B Mohr (Paul Siebeck). 
Roell, W. (1987): Apokalyptiker und Integrierte. Bemerkungen zur institutionalisierten Freudschen Psychoanalyse in Zürich, Journal, Psychoanalytisches Seminar Zürich, Sondernummer: 35-38.

Rothschild, B. (1988): Einführung in die Idiotenproblematik, Journal, Psychoanalytisches Seminar Zürich, Nr. 18: 8-10.

Rothschild, B. (1993): «Plataforma» in den letzten zwanzig Jahren. Vortrag anlässlich des 20. Jubiläums ihrer Gründung, in: Luzifer-Amor, 6. Jahrgang, Heft 12: 55-62.

Rothschild, B. (2000): Psychoanalyse-mündliche Tradierung-Selbstmystifizierung, Journal, Psychoanalytisches Seminar Zürich, Nr. 38: 5-16 (Neudruck aus

Psychoanalytische Blätter, 1996: 7-34).

Tocqueville, A. de (1968): De la démocratie en amérique. Paris, Gallimard.

Trotha, T. von (1994): Koloniale Herrschaft. Zur soziologischen Theorie der

Staatsentstehung am Beispiel des «Schutzgebietes Togo», Tübingen: J.C.B. Mohr (Paul Siebeck).

Valk, J. (1987):Verlust der Institution -Verlust der historischen Kontinuität? Journal, Psychoanalytisches Seminar Zürich, Sondernummer: 7-8.

Weilenmann, M. (1996): Das Faschismus-«Syndrom». Einige Gedanken zum Untersuchungsgegenstand, in: Journal-Sondernummer: Das Faschismus«Syndrom», Psychoanalytisches Seminar Zürich, Sondernummer: 7-12.

Weilenmann, M. (2000): Gefangen zwischen den «fünf Fingern der linken Hand»? Einige kritische Gedanken zur Geschichte der Entpolitisierung am Psychoanalytischen Seminar Zürich, Journal, Psychoanalytisches Seminar Zürich, Nr. 39: 6-21.

\section{Anmerkungen}

1 Leicht überarbeitete und gekürzte Fassung eines Artikels, der von mir unter demselben Obertitel bereits im Journal 39 erschienen ist (vgl. Weilenmann, 2000).

2 Unter dem Übernamen «Frösche» figurierten damals die Autoren der «Selnaustrasse», Jaron Bendkower, Karin Merzbacher, Thomas Kappeler und Daniel Strassberg. Ihr Artikel trägt den Titel «Von den Schwierigkeiten, die Regressionen der Couch in einer demokratischen Couch-Institution zu überwinden - oder: Die Tellstrasse aus der Froschperspektive», 1980, mutmaßliche Seiten 2-6 (Seitenzahlen gab es im damaligen Journal noch nicht).

3 Details dazu vgl. Emilio Modena in dieser Nummer.

$4 \quad$ Hinter der Couch sollte subtil, professionell und analytisch gearbeitet werden; vor und außerhalb der Couch - also im politischen Feld - so interventionistisch, wie es unsere Fähigkeiten gestatten.

5 Zu nennen sind hier die späteren Werke von Paul Parin wie: «Untrügliche Zeichen von Veränderung» (1982), «Zu viele Teufel im Land» (1985), «Noch ein Leben» (1990) oder «Eine Sonnenuhr für beide Hemisphären» (1995). 
6 Vgl. dazu die Arbeiten von Fritz Morgenthaler wie: «Technik. Zur Dialektik der psychoanalytischen Praxis» (1978), «Homosexualität, Heterosexualität, Perversion» (1984), «Der Traum» (1986); von Paul Parin «Der Widerspruch im Subjekt» (1978) und von Paul und Goldy Parin-Matthèy «Subjekt im Widerspruch» (1986)

$7 \quad$ Eine solche Dichotomisierung erweckt unter anderem den Anschein, politische Herrschaft, also die Kombination von Macht, Ausdehnung und Zwang sei nicht triebmotiviert und Kultur habe nur etwas mit Ordnung, also mit Normativität zu tun.

$8 \quad$ Natürlich lässt sich diese Entwicklung auch vor dem Hintergrund des damals noch virulenten Kalten Krieges, der unselig machenden Polarisierungen in der schweizerischen Politik (Diamantfeiern) und der Jugendunruhen lesen. Zugleich enthalten solche Bilder aber auch eine Botschaft an die Diskursteilnehmer des Verhältnisses Psychoanalyse-Gesellschaftskritik.

9 Das PSZ war zu keinem Zeitpunkt keine Institution. Nach dem bekannten Soziologen René König spricht man von einer Institution immer dann, «wenn es um Komplexe von institutionalisierten Rollenintegraten oder Statusbeziehungen geht, die in einem gegebenen sozialen System von «strategischer Bedeutung,(Talcott Parsons) sind. (...) Von strategischer Bedeutung eines Verhaltens oder von Rollen und Statusbeziehungen kann man dann sprechen, wenn es sich um allgemein interessierende Beziehungen handelt»(1958/79:145). Darunter fallen im vorliegenden Zusammenhang nicht nur organisatorische Begriffe wie die «TV», die «Seminarleitung» oder die «Supervisorenliste», sondern auch weichere Merkmale gehören dazu wie etwa die «Selbstdeklaration», die Frage, «wer bei wem analysiert wurde und wer bei wem in Supervision geht» (kann einen Einfluss auf das analytische Denken haben), die «Settingkonstruktion» (die als normative Struktur Psychoanalyse erst ermöglicht), die Durchführung von öffentlichen Veranstaltung und Kursen (Versuch derVerankerung vonWelt-Sichten im öffentlichen Raum/ Prestige), Fragen nach der internen Distribution von Patienten (Ausgestaltung der inneren Netzwerke, der so genannten «Kränzlis» = wirtschaftliche und innenpolitische Machtgruppen), das Ruffördernde und/oder Rufschädigende Buschtelephon etc.

10 Vgl. dazu insbesondere den erwähnten Artikel der «Frösche» in Journal Nr. 1

11 Cremerius behauptet zum Beispiel keck, die Entfaltung wissenschaftlichen Denkens würde durch die Schaffung institutioneller Strukturen behindert, wo doch die gesamte politische Geschichte zeigt, wie die Entwicklung wissenschaftlicher Leistungen sehr eng mit der Bildung hochkomplexer Gesellschaften, namentlich der Geschichte der Bürokratie zusammenhängt (was immer das - auch für das institutionalisierte Verfahren der Psychoanalyse - heißen mag).

12 Vgl. dazu auch den meines Erachtens sehr überzeugenden Beitrag von Berthold Rothschild im Journal Nr. 38, 2000: 5-16

13 Berthold Rothschild verwendet diesbezüglich den Begriff der «Oral History», den er mit der Entwicklung einer Binnenmoral, so genannten «Salbungs-Ketten» (Wer wurde von wem analysiert und supervidiert?) und einer wachsenden Selbstmystifizierung von Psychoanalyse verknüpft (vgl. Journal 38, 2000: 5-16)

14 Hypothetisch könnte man z. B. annehmen, dass eine verstärkte Auseinandersetzung mit der Bildung informeller Herrschaftssysteme auch den Blick «nach innen», in die Innereien des PSZ verändern würde (passt doch der real praktizierte freie Kapitalismus schlecht zum linken Selbstbild) und dass diese Auseinandersetzung zum Teil gerade nicht gesucht wird, weil damit das in Abgrenzung zu anderen Instituten verwendete Bild vom «(linken) selbstverwalteten PSZ» eine feudal-kapitalistische Note bekäme (vgl. etwa den von Emilio Modena in dieser Nummer publizierten Artikel zur Geschichte des selbstverwalteten PSZ. Berthold Rothschild erwähnt im Zusammenhang der verschiedenen Abhängigkeiten in psychoanalytischen Instituten den Begriff der «Salbungs-Ketten», der an recht intakte Klientelsysteme erinnert, über «bloß» feudalistische Abhängigkeiten aber hinausgeht, weil die Abhängigkeit nicht nur pekuniär-machtpolitischer sondern auch emotional-intellektueller Art ist).

15 ... wie heißt es doch: «Geb’ ich Dir den kleinen Finger - willst Du die ganze Hand!» 
16 ...wobei anzumerken ist, dass «Staatsstreiche» gewöhnlich von Diktatoren ausgeführt werden ...

17 Die Abspaltungsgeschichte machte alle betroffen und erschwerte ganz besonders die Distanz, die notwendig wäre, um die sich abspielenden Prozesse der Machtbildung (vgl. z. B. Popitz 1968) auch angemessen untersuchen zu können. Wer sich jedoch stattdessen auf Betroffenheit spezialisiert und Parteilichkeit als Fachkompetenz ausgegeben hat, diskreditierte nicht nur psychoanalytisches Denken sondern Denken schlechthin.

$18 \ldots$ etwa zu einem methodischen Problem, nämlich dass nicht nur die Analyse psychischer Prozesse sondern auch die Analyse politischer Prozesse ihr jeweils eigenes Setting erforderte; oder dazu, dass die analytische Arbeit im politischen Feld ein Setting voraussetzte, das jenseits des Parteiengezänks zu liegen kommt, weil es nur so möglich ist, den Kampf um die unterschiedlichen Wirklichkeitsdefinitionen auch konzeptionell zu fassen; oder dazu, dass es, wie sich Georges Devereux (1978: 117) ausdrückte: «(..) auf keinen Fall zulässig (ist), ein sich aufdas Individuum beziehendes Begriffsmodell aufdas soziokulturelle System als Ganzes zu übertragen und soziokulturelle Strukturen und Prozesse rein im Rahmen der Psychologie des Individuums zu interpretieren, auch wenn dieses Individuum zu der Gesellschaft gehört, deren Struktur und Prozess auf diese Weise (miss-)interpretiert wird. Genauer und in einfacheren Worten: die Verfassung der Bundesrepublik Deutschland ist und kann nie das Über-Ich oder Ich-Ideal der bundesdeutschen Gesellschaft sein (...) aus dem guten und hinreichenden Grund, dass eine Gesellschaft kein Über-Ich oder Ich-Ideal hat, wie auch die Psyche eines Individuums keine Verfassung und kein Bundesverfassungsgericht hat» usw.

19 Auch wenn der Artikel vor 20 Jahren geschrieben wurde, dennoch Hand aufs Herz: Ist jemand, der mir, sagen wir, eine Neidproblematik deutet, «links»? Ist jemand, der die psychoanalytischen Konzepte "in schärfere dialektische Begriffe fasst»(ebenda) und den «historischen Zusammenhang (mitdenkt)»(ebenda) «links»? Sind Personen, die historische Zusammenhänge mitdenken «links»? Reduziert sich politisches Denken auf die Anwendung der dialektisch-materialistischen Methode, oder geht es vielleicht nicht doch um ein klein wenig etwas anderes, nämlich primär um dieWahrnehmung und Analyse eines eigengesetzlichen Interaktionsgeflechtes außerhalb von Chaiselongue und Couch? Gibt es - jenseits der politischen Debatten - eine Art psychoanalytische Binnenlogik des «Links-Seins»? (>ideologische Basis)

20 ... die alle in einem äußerst gebrochenen, nicht kausalen Verhältnis zu den politischen Machtbildungsprozessen stehen / MW ...

21 Bei aller Achtung gegenüber den Leistungen von Sigmund Freud sei an dieser Stelle auch einmal gesagt, dass der Begründer der Psychoanalyse nicht wegen seiner gesellschaftswissenschaftlichen Beiträge berühmt wurde. Ohne Beizug nicht-psychoanalytischer Theorien und ohne den Rekurs auf Erfahrungen, die gerade nicht nur hinter der Couch gemacht werden, ist in diesem komplexen Feld nichts zu haben. Das machen nicht zuletzt auch die herausragenden Arbeiten Paul Parins (1978) oder Mario Erdheims (1982) deutlich.

22 Im Nachgang zum so genannten «Interlakener Lehrstück» hieß es noch: «Merke: Mit der Psychoanalyse allein versteht man die psychoanalytischen Gesellschaften nie» (zit. $\mathrm{n}$. Emilio Modena, in dieser Nummer).

23 Vgl. dazu die Beiträge von Danielle Bazzi, Judith Valk, Heinz-Peter Müller, Jarow Bendkower, Pedro Grosz, Madeleine Dreyfus und Wolfgang Roell (alle 1987). Einzig der Beitrag von Emilio Modena greift nochmals Teile der alten gemeinsamen Utopie auf, versucht sich dem ideologisch motivierten Sog der egozentrischen Identitätsfrage zu entziehen, die Innenansichten an einer Außenperspektive zu brechen und darauf zu dringen, dass sich «das Politische aus der Psychoanalyse zu verflüchtigen droht» (1987: 35).

24 Damit, mit dem «aufs Dach bekommen», hat Ralph Binswanger als Leiter des «Revolutionären Aufbau Zürich» ohnehin seine Erfahrungen; auch darf vermutet werden, dass er auch ohne Psychoanalyse im politischen Kampf hin und wieder eins aufs Dach bekommt - so what? 
25 ... etwa indem im öffentlichen Raum mit den Begriffen der psychoanalytischen Topik hantiert wird ...

26 Näheres dazu vgl. Weilenmann, Markus: Das Faschismus-«Syndrom». Einige Gedanken zum Untersuchungsgegenstand, in: Journal-Sondernummer: Das Faschismus-«Syndrom», 1996: $11-12$

27 Das setzt aber auch die Aneignung eines anderen, gesellschaftswissenschaftlichen Spezial-wissens voraus, womit wir wieder bei der Kritik der «Frösche» angelangt wären, nämlich dass es am PSZ bis zum heutigen Tag an einem entsprechenden Lehrangebot mangelt. Wohl wird im neuen Weiterbildungskonzept des Seminars «Gesellschafts-, Kultur- und Wissenschaftstheorie» als einer von sechs Sektoren aufgeführt, aus denen Kurse besucht werden müssen, um sich das theoretische Rüstzeug in Psychoanalyse anzueignen. Es geht aber um die Umsetzung dieses Rahmens und dies-bezüglich liegt am PSZ - abgesehen von der soeben durchgeführten Institutionsanalyse - noch einiges im Argen. Insbesondere fehlt es an verbindlichen und gesellschaftswissenschaftlich vertretbaren Kriterien, die darüber entscheiden, ob ein Kursangebot in diesen Sektor fällt oder nicht. Das gesellschaftswissenschaftliche Kursangebot riskiert vielmehr zu einer Residualkategorie zu verkommen, in welcher allerlei Kurse zur Geschichte von Psychoanalyse angeboten werden.

28 ... man könnte sich auch einmal darüber unterhalten, weshalb immerzu nur die perverse, gewalttätige Seite der Kultur hervorgehoben werden muss oder weshalb die Relevanz bspw. der politischen Psychologie so häufig aus gesellschaftlichen Horrorszenarien abgeleitet wird (so etwa Busch, 2000) und weshalb der Grad an sexueller Wunschbefriedigung, der in ihr auch enthalten ist, so gar nicht zur Sprache kommt, vgl. Strafrecht, Vatermord, Totem und Tabu usw.

29 ... obwohl es selbstverständlich auch eine Kulturgeschichte des Wunsches gibt, die, auch wenn sie sich aus der menschlichen Subjektivität ergibt, anderen, nämlich interaktiven, interpsychischen, symbolischen, machtpolitisch und geschichtlich begründeten Abläufen folgt als denjenigen der subjektiven Wunschbildung in einem einzelnen Subjekt, die sich aus den intrapsychischen Triebkonflikten und dem daraus resultierenden Begehren herleiten $\ldots$

30 Sie eignete sich allenfalls dazu, das gesellschaftliche Phänomen der Ehre, des sozialen Prestiges u. a. einem zweiten, psychoanalytischen Sinn zuzuführen.

31 Modena, Emilio (2000b): Aggredo und Libido. Zur Entmythologisierung der Freudschen Triebtheorie. Psychosozial, Nr. 84: 15-55.

32 Vgl. Knellessen/Passett/Schneider (1995): DieVerflüchtigung undWiedergewinnung des Politischen, in: Jourbal Nr. 30: 22-47.

33 ... etwa in Abgrenzungzum Akteur-Struktur-Paradigma von Bourdieu (1979) und Giddens (1984). So bemängelt etwa von Trotha (1994: 7), dass «dieWirklichkeit des «neuen soziologischen Akteurs eine merkwürdig emotionslose Welt (ist), in der das Drama der Vergesellschaftung zur strategischen Kalkulation und zum manipulativen Einsatz der Ressourcen von Akteuren wird, die innerhalb einer Struktur alternativerWege Optionen wählen, die ihnen den größten Nutzen versprechen.»

$34 \quad$... weshalb ja auch mit Recht sich allenfalls aufdrängende Modifikationen der Absichtserklärung äußerst behutsam angegangen werden ... 